\title{
The Simplest Equation Method and Its Application for Solving the Nonlinear NLSE, KGZ, GDS, DS, and GZ Equations
}

\author{
Yun-Mei Zhao, Ying-Hui He, and Yao Long \\ Department of Mathematics, Honghe University, Mengzi, Yunnan 661100, China \\ Correspondence should be addressed to Yun-Mei Zhao; zhaoyunmei2000@126.com
}

Received 17 September 2013; Revised 7 November 2013; Accepted 7 November 2013

Academic Editor: Anjan Biswas

Copyright (c) 2013 Yun-Mei Zhao et al. This is an open access article distributed under the Creative Commons Attribution License, which permits unrestricted use, distribution, and reproduction in any medium, provided the original work is properly cited.

\begin{abstract}
A good idea of finding the exact solutions of the nonlinear evolution equations is introduced. The idea is that the exact solutions of the elliptic-like equations are derived using the simplest equation method and the modified simplest equation method, and then the exact solutions of a class of nonlinear evolution equations which can be converted to the elliptic-like equation using travelling wave reduction are obtained. For example, the perturbed nonlinear Schrödinger's equation (NLSE), the Klein-Gordon-Zakharov (KGZ) system, the generalized Davey-Stewartson (GDS) equations, the Davey-Stewartson (DS) equations, and the generalized Zakharov (GZ) equations are investigated and the exact solutions are presented using this method.
\end{abstract}

\section{Introduction}

Nonlinear phenomena exist in all areas of science and engineering, such as fluid mechanics, plasma physics, optical fibers, biology, solid state physics, chemical kinematics, and chemical physics. It is well known that many nonlinear partial differential equations (NLPDEs) are widely used to describe these complex physical phenomena. The exact solution of a differential equation gives information about the construction of complex physical phenomena. Therefore, seeking exact solutions of NLPDEs has long been one of the central themes of perpetual interest in mathematics and physics. With the development of symbolic computation packages, like Maple and Mathematica, many powerful methods for finding exact solutions have been proposed, such as the homogeneous balance method $[1,2]$, the auxiliary equation method [3], the sine-cosine method [4], the Jacobi elliptic function method [5], the exp-function method [6], the tanhfunction method $[7,8]$, the Darboux transformation $[9,10]$, and the $\left(G^{\prime} / G\right)$-expansion method $[11,12]$.

The simplest equation method is a very powerful mathematical technique for finding exact solutions of nonlinear ordinary differential equations. It has been developed by Kudryashov $[13,14]$ and used successfully by many authors for finding exact solutions of ODEs in mathematical physics [15-19].
In this paper, we first apply the simplest equation method and the modified simplest equation method to derive the exact solutions of the elliptic-like equation, and then the exact solutions of a class of nonlinear evolution equations which can be converted to the elliptic-like equation using travelling wave reduction are obtained.

\section{Description of Methods}

\subsection{The Simplest Equation Method}

Step 1. Suppose that we have a nonlinear partial differential equation (PDE) for $u(x, t)$ in the form

$$
N\left(u, u_{t}, u_{x}, u_{t t}, u_{x t}, u_{x x}, \ldots\right)=0 \text {, }
$$

where $N$ is a polynomial in its arguments.

Step 2. By taking $u(x, t)=u(\xi), \xi=x-c t$, we look for traveling wave solutions of (1) and transform it to the ordinary differential equation (ODE)

$$
N\left(u,-c u^{\prime}, u^{\prime}, c^{2} u^{\prime \prime},-c u^{\prime \prime}, u^{\prime \prime}, \ldots\right)=0
$$


Step 3. Suppose the solution $u$ of (2) can be expressed as a finite series in the form

$$
u=\sum_{i=0}^{n} A_{i}(H(\xi))^{i},
$$

where $H(\xi)$ satisfies the Bernoulli or Riccati equation, $n$ is a positive integer that can be determined by balancing procedure, and $A_{i}(i=0,1,2, \ldots, n)$ are parameters to be determined.

The Bernoulli equation we consider in this paper is

$$
H^{\prime}(\xi)=a H(\xi)+b H^{2}(\xi),
$$

where $a$ and $b$ are constants. Its solutions can be written as

$$
\begin{aligned}
H(\xi) & =\frac{-a C_{1}}{b\left(C_{1}+\cosh \left(a\left(\xi+\xi_{0}\right)\right)-\sinh \left(a\left(\xi+\xi_{0}\right)\right)\right)}, \\
H(\xi) & =\frac{-a\left(\cosh \left(a\left(\xi+\xi_{0}\right)\right)+\sinh \left(a\left(\xi+\xi_{0}\right)\right)\right)}{b\left(C_{2}+\cosh \left(a\left(\xi+\xi_{0}\right)\right)+\sinh \left(a\left(\xi+\xi_{0}\right)\right)\right)},
\end{aligned}
$$

where $C_{1}, C_{2}$, and $\xi_{0}$ are constants.

For the Riccati equation

$$
H^{\prime}(\xi)=a H^{2}(\xi)+b H(\xi)+s,
$$

where $a, b$, and $s$ are constants, we will use the solutions

$$
\begin{gathered}
H(\xi)=-\frac{b}{2 a}-\frac{\theta}{2 a} \tanh \left[\frac{\theta}{2}\left(\xi+\xi_{0}\right)\right], \\
H(\xi)=-\frac{b}{2 a}-\frac{\theta}{2 a} \tanh \left(\frac{\theta}{2} \xi\right) \\
+\frac{\operatorname{sech}(\theta \xi / 2)}{C \cosh (\theta \xi / 2)-(2 a / \theta) \sinh (\theta \xi / 2)},
\end{gathered}
$$

where $\theta^{2}=b^{2}-4 a s$.

Step 4. Substituting (3) into (2) with (4) (or (6)), then the left hand side of $(2)$ is converted into a polynomial in $H(\xi)$; equating each coefficient of the polynomial to zero yields a set of algebraic equations for $A_{i}, a, b(i=0,1,2, \ldots, n)$. Solving the algebraic equations by symbolic computation, we can determine those parameters explicitly.

Step 5. Assuming that the constants $A_{i}, a, b(i=0,1,2, \ldots$, $n$ ) can be obtained in Step 4 and substituting the results into (3), then we obtain the exact traveling wave solutions for (1).

2.2. The Modified Simplest Equation Method. In the modified version, one makes an ansatz for the solution $u(\xi)$ as

$$
u=\sum_{i=0}^{n} a_{i}\left(\frac{\psi^{\prime}(\xi)}{\psi(\xi)}\right)^{i}
$$

where $a_{i}(i=0,1,2, \ldots, n)$ are arbitrary constants to be determined, such that $a_{n} \neq 0$ and $\psi(\xi)$ is an unspecified function to be determined afterward.
Substitute (8) into (2) and then we account the function $\psi(\xi)$. As a result of this substitution, we get a polynomial of $\psi^{\prime}(\xi) / \psi(\xi)$ and its derivatives. In this polynomial, we equate the coefficients of the same power of $\psi^{-j}(\xi)$ to zero, where $j \geq$ 0 . This procedure yields a system of equations which can be solved to find $a_{i}(i=0,1,2, \ldots, n), \psi(\xi)$, and $\psi^{\prime}(\xi)$. Then the substitution of the values of $a_{i}(i=0,1,2, \ldots, n), \psi(\xi)$, and $\psi^{\prime}(\xi)$ into (8) completes the determination of exact solutions of (1).

\section{Solutions of the Elliptic-Like Equation}

Now, let us choose the following elliptic-like equation

$$
A \phi^{\prime \prime}(\xi)+B \phi(\xi)+C \phi^{3}(\xi)=0
$$

where $A, B$, and $C$ are arbitrary constants. Equation (9) is one of the most important auxiliary equations, because many nonlinear evolution equations can be converted to (9) using the travelling wave reduction.

\subsection{Using Simplest Equation Method}

3.1.1. Solutions of (9) Using the Bernoulli Equation as the Simplest Equation. Considering the homogeneous balance between $\phi^{\prime \prime}(\xi)$, and $\phi^{3}(\xi)$ we get $n=1$, so the solution of (9) is the form

$$
\phi(\xi)=A_{0}+A_{1} H(\xi)
$$

Substituting (10) into (9) and making use of the Bernoulli equation (4) and then equating the coefficients of the functions $H^{i}(\xi)$ to zero, we obtain an algebraic system of equations in terms of $A_{i}(i=0,1), a$, and $b$. Solving this system of algebraic equations, with the aid of Maple, we obtain

$$
\begin{array}{lll}
A_{0}= \pm i \sqrt{\frac{B}{C}}, & A_{1}= \pm i \sqrt{\frac{2 A}{C}}, & a=\sqrt{\frac{2 B}{A}}, \\
A_{0}= \pm i \sqrt{\frac{B}{C}}, & A_{1}=\mp i \sqrt{\frac{2 A}{C}}, & a=-\sqrt{\frac{2 B}{A}} .
\end{array}
$$

Therefore, using solutions (5) of (4) and ansatz (10), we obtain the following exact solution of (9):

$$
\begin{aligned}
\phi_{1}(\xi)= \pm i \sqrt{\frac{B}{C}}(1- & \left(2 C_{1}\right) \\
\times & \left(C_{1}+\cosh \left(\sqrt{\frac{2 B}{A}}\left(\xi+\xi_{0}\right)\right)\right. \\
& \left.\left.-\sinh \left(\sqrt{\frac{2 B}{A}}\left(\xi+\xi_{0}\right)\right)\right)^{-1}\right),
\end{aligned}
$$




$$
\begin{aligned}
& \phi_{2}(\xi)= \pm i \sqrt{\frac{B}{C}}\left(1-\left(2 C_{1}\right)\right. \\
& \times\left(C_{1}+\cosh \left(\sqrt{\frac{2 B}{A}}\left(\xi+\xi_{0}\right)\right)\right. \\
& \left.\left.+\sinh \left(\sqrt{\frac{2 B}{A}}\left(\xi+\xi_{0}\right)\right)\right)^{-1}\right) \\
& \phi_{3}(\xi)= \pm i \sqrt{\frac{B}{C}}\left(1-\left(2 \left(\cosh \left(\sqrt{\frac{2 B}{A}}\left(\xi+\xi_{0}\right)\right)\right.\right.\right. \\
& \left.\left.+\sinh \left(\sqrt{\frac{2 B}{A}}\left(\xi+\xi_{0}\right)\right)\right)\right) \\
& \times\left(C_{2}+\cosh \left(\sqrt{\frac{2 B}{A}}\left(\xi+\xi_{0}\right)\right)\right. \\
& \left.\left.+\sinh \left(\sqrt{\frac{2 B}{A}}\left(\xi+\xi_{0}\right)\right)\right)^{-1}\right) \\
& \phi_{4}(\xi)= \pm i \sqrt{\frac{B}{C}}\left(1-\left(2 \left(\cosh \left(\sqrt{\frac{2 B}{A}}\left(\xi+\xi_{0}\right)\right)\right.\right.\right. \\
& \left.\left.-\sinh \left(\sqrt{\frac{2 B}{A}}\left(\xi+\xi_{0}\right)\right)\right)\right) \\
& \times\left(C_{2}+\cosh \left(\sqrt{\frac{2 B}{A}}\left(\xi+\xi_{0}\right)\right)\right. \\
& \left.\left.-\sinh \left(\sqrt{\frac{2 B}{A}}\left(\xi+\xi_{0}\right)\right)\right)^{-1}\right) .
\end{aligned}
$$

3.1.2. Solutions of (9) Using Riccati Equation as the Simplest Equation. Suppose the solutions of (9) are the form

$$
\phi(\xi)=B_{0}+B_{1} H(\xi) .
$$

Substituting (13) into (9) and making use of the Riccati equation (6) and then equating the coefficients of the functions $H^{i}(\xi)$ to zero, we obtain an algebraic system of equations in terms of $B_{i}(i=0,1), a, b$, and $s$. Solving this system of algebraic equations, with the aid of Maple, one possible set of values of $B_{i}(i=0,1), a, b$, and $s$ is

$$
B_{0}= \pm i \sqrt{\frac{A}{2 C}} b, \quad B_{1}= \pm i \sqrt{\frac{2 A}{C}} a, \quad s=\frac{A b^{2}-2 B}{4 A a} \text {. }
$$

Therefore, using solutions (7) of (6) and ansatz (13), we obtain the following exact solution of (9):

$$
\begin{array}{r}
\phi_{5}(\xi)=\mp i \sqrt{\frac{B}{C}} \tanh \left(\sqrt{\frac{B}{2 A}}\left(\xi+\xi_{0}\right)\right) \\
\phi_{6}(\xi)=\mp i \sqrt{\frac{B}{2 C}}\left(\left(C \sqrt { \frac { 2 B } { A } } \operatorname { s i n h } \left(\sqrt{\left.\frac{B}{2 A} \xi\right)}\right.\right.\right. \\
\left.-2 a \cosh \left(\sqrt{\frac{B}{2 A} \xi}\right)\right) \\
\times\left(C \sqrt{\frac{B}{A}} \cosh \left(\sqrt{\frac{B}{2 A}} \xi\right)\right. \\
\left.\left.-a \sqrt{2} \sinh \left(\sqrt{\frac{B}{2 A}} \xi\right)\right)^{-1}\right) .
\end{array}
$$

3.2. Using Modified Simplest Equation Method. Suppose the solution of (9) is the form

$$
\phi(\xi)=a_{0}+a_{1}\left(\frac{\psi^{\prime}(\xi)}{\psi(\xi)}\right)
$$

where $a_{0}$ and $a_{1}$ are constants, such that $a_{1} \neq 0$, and $\phi(\xi)$ is an unspecified function to be determined. It is simple to calculate that

$$
\begin{gathered}
\phi^{\prime}=a_{1}\left(\frac{\psi^{\prime \prime}}{\psi}-\left(\frac{\psi^{\prime}}{\psi}\right)^{2}\right), \\
\phi^{\prime \prime}=a_{1}\left(\frac{\psi^{\prime \prime \prime}}{\psi}\right)-3 a_{1}\left(\frac{\psi^{\prime \prime} \psi^{\prime}}{\psi^{2}}\right)+2 a_{1}\left(\frac{\psi^{\prime}}{\psi}\right)^{3}, \\
\phi^{3}=a_{1}^{3}\left(\frac{\psi^{\prime}}{\psi}\right)^{3}+3 a_{1}^{2} a_{0}\left(\frac{\psi^{\prime}}{\psi}\right)^{2}+3 a_{1} a_{0}^{2}\left(\frac{\psi^{\prime}}{\psi}\right)+a_{0}^{3} .
\end{gathered}
$$

Substituting the values of $\phi, \phi^{\prime \prime}$, and $\phi^{3}$ into (9) and equating the coefficients of $\psi^{0}, \psi^{-1}, \psi^{-2}$, and $\psi^{-3}$ to zero yield

$$
\begin{gathered}
\psi^{0}: C a_{0}^{3}+B a_{0}=0, \\
\psi^{-1}:\left(3 C a_{0}^{2}+B\right) \psi^{\prime}+A \psi^{\prime \prime \prime}=0, \\
\psi^{-2}:-3 A \psi^{\prime \prime}+3 C a_{0} a_{1} \psi^{\prime}=0, \\
\psi^{-3}:\left(C a_{1}^{3}+2 A a_{1}\right)\left(\psi^{\prime}\right)^{3}=0 .
\end{gathered}
$$

Solving (18), we obtain

$$
a_{0}=0, \quad a_{0}= \pm i \sqrt{\frac{B}{C}} .
$$

And solving (21), we obtain

$$
a_{1}= \pm i \sqrt{\frac{2 A}{C}}, \quad \text { since } a_{1} \neq 0 .
$$


Case 1. When $a_{0}=0$, we obtain trivial solution; therefore, the case is rejected.

Case 2. When $a_{0}= \pm i \sqrt{B / C}$ and $a_{1}= \pm i \sqrt{2 A / C}$, (19) and (20) yield

$$
\frac{\psi^{\prime \prime \prime}}{\psi^{\prime \prime}}+\sqrt{\frac{2 B}{A}}=0
$$

Integrating (24) with respect to $\xi$, we obtain

$$
\psi^{\prime \prime}=C_{1} \exp \left(-\sqrt{\frac{2 B}{A}} \xi\right) \text {. }
$$

Using (25), from (20), we obtain

$$
\psi^{\prime}=-\sqrt{\frac{A}{2 B}} C_{1} \exp \left(-\sqrt{\frac{2 B}{A}} \xi\right) .
$$

Upon integration, we obtain

$$
\psi=\frac{A C_{1}}{2 B} \exp \left(-\sqrt{\frac{2 B}{A}} \xi\right)+C_{2},
$$

where $C_{1}$ and $C_{2}$ are constants of integration. Therefore, the exact solution of (9) is

$$
\phi_{7}(\xi)= \pm i \sqrt{\frac{B}{C}}\left(1-\frac{2 A C_{1} \exp (-\sqrt{(2 B / A)} \xi)}{A C_{1} \exp (-\sqrt{(2 B / A)} \xi)+2 B C_{2}}\right) .
$$

From (28), we obtain the exact solution of (9) which is

$$
\begin{aligned}
\phi_{8}(\xi)= \pm i \sqrt{\frac{B}{C}}(1 & -\left(2 A C _ { 1 } \left(\cosh \left(\sqrt{\frac{B}{2 A} \xi}\right)\right.\right. \\
& \left.\left.-\sinh \left(\sqrt{\frac{B}{2 A} \xi}\right)\right)\right) \\
\times & \left(\left(2 B C_{2}+A C_{1}\right) \cosh \left(\sqrt{\frac{B}{2 A} \xi}\right)\right. \\
& \left.\left.+\left(2 B C_{2}-A C_{1}\right) \sinh \left(\sqrt{\frac{B}{2 A}} \xi\right)\right)^{-1}\right) .
\end{aligned}
$$

We can arbitrarily choose the parameters $C_{1}$ and $C_{2}$. Therefore, if we set $C_{1}=2 B C_{2} / A,(29)$ reduces to

$$
\phi_{9}(\xi)= \pm i \sqrt{\frac{B}{C}} \tanh \left(\sqrt{\frac{B}{2 A}} \xi\right) .
$$

Again setting $C_{1}=-\left(2 B C_{2} / A\right),(29)$ reduces to,

$$
\phi_{10}(\xi)= \pm i \sqrt{\frac{B}{C}} \operatorname{coth}\left(\sqrt{\frac{B}{2 A}} \xi\right) .
$$

Using hyperbolic function identities, from (30) and (31), we obtain the following periodic solutions

$$
\begin{aligned}
& \phi_{11}(\xi)= \pm \sqrt{\frac{B}{C}} \tan \left(\sqrt{\frac{-B}{2 A}} \xi\right), \\
& \phi_{12}(\xi)= \pm \sqrt{\frac{B}{C}} \cot \left(\sqrt{\frac{-B}{2 A}} \xi\right) .
\end{aligned}
$$

\section{Exact Solutions of Some Class of NLPDEs}

4.1. The Perturbed Nonlinear Schrödinger's Equation (NLSE) in the Form [20]. Using

$$
\begin{aligned}
i u_{t}+ & u_{x x}+\alpha u|u|^{2} \\
& +i\left[\gamma_{1} u_{x x x}+\gamma_{2}|u|^{2} u_{x}+\gamma_{3} u\left(|u|^{2}\right)_{x}\right]=0
\end{aligned}
$$

where $\gamma_{1}$ is the third order dispersion, $\gamma_{2}$ is the nonlinear dispersion, while $\gamma_{3}$ is also a version of nonlinear dispersion. We assume that (33) has exact solution in the form

$$
u(x, t)=\phi(\xi) \exp (i(\lambda x-\omega t)), \quad \xi=k(x-c t),
$$

where $\lambda, \omega, k$, and $c$ are arbitrary constant to be determined. Substituting (34) into (33), removing the common factor $\exp (i(\lambda x-\omega t))$, we have

$$
\begin{aligned}
& i\left(\gamma_{1} k^{3} \phi^{\prime \prime \prime}-k\left(c-2 \lambda+3 \gamma_{1} \lambda^{2}\right) \phi^{\prime}+k\left(\gamma_{2}+2 \gamma_{3}\right) \phi^{2} \phi^{\prime}\right) \\
& +k^{2}\left(1-3 \gamma_{1} \lambda\right) \phi^{\prime \prime}+\left(\omega-\lambda^{2}+\gamma_{1} \lambda^{3}\right) \phi \\
& +\left(\alpha-\gamma_{2} \lambda\right) \phi^{3}=0,
\end{aligned}
$$

where $\gamma_{i}(i=1,2$, and 3$), \alpha$, and $k$ are positive constants and the prime means differentiation with respect to $\xi$. Then we have two equations as follows

$$
\begin{aligned}
& \gamma_{1} k^{2} \phi^{\prime \prime \prime}-\left(c-2 \lambda+3 \gamma_{1} \lambda^{2}\right) \phi^{\prime}+\left(\gamma_{2}+2 \gamma_{3}\right) \phi^{2} \phi^{\prime}=0, \\
& k^{2}\left(1-3 \gamma_{1} \lambda\right) \phi^{\prime \prime}+\left(\omega-\lambda^{2}+\gamma_{1} \lambda^{3}\right) \phi+\left(\alpha-\gamma_{2} \lambda\right) \phi^{3}=0 .
\end{aligned}
$$

Integrating (36) with respect to $\xi$ once and setting the integration constant to be zero, then we have

$$
\gamma_{1} k^{2} \phi^{\prime \prime}+\left(2 \lambda-c-3 \gamma_{1} \lambda^{2}\right) \phi+\left(\frac{1}{3} \gamma_{2}+\frac{2}{3} \gamma_{3}\right) \phi^{3}=0 .
$$

As (37) and (38) have the same solutions, we have the following equation:

$$
\frac{\gamma_{1}}{1-3 \gamma_{1} \lambda}=\frac{2 \lambda-c-3 \gamma_{1} \lambda^{2}}{\omega-\lambda^{2}+\gamma_{1} \lambda^{3}}=\frac{C}{\alpha-\gamma_{2} \lambda},
$$

where $C=(1 / 3) \gamma_{2}+(2 / 3) \gamma_{3}$.

From (39), we can obtain

$$
\begin{array}{r}
\omega=\frac{\left(\alpha-\gamma_{2} \lambda\right)\left(2 \lambda-c-3 \gamma_{1} \lambda^{2}\right)}{C}+\lambda^{2}-\gamma_{1} \lambda^{3}, \\
\lambda=\frac{C-\alpha \lambda_{1}}{3 C \gamma_{1}-\gamma_{1} \gamma_{2}} .
\end{array}
$$


Based on the conclusion just mentioned, we only solve (38) or (37), instead of both (37) and (38), provided that (37) and (36) are replaced by (40), respectively, we get

$$
A \phi^{\prime \prime}(\xi)+B \phi(\xi)+C \phi^{3}(\xi)=0 .
$$
ed by

Equation (41) is identical to (9) and $A, B$, and $C$ are defin-

$$
A=\gamma_{1} k^{2}, \quad B=2 \lambda-c-3 \gamma_{1} \lambda^{2}, \quad C=\frac{1}{3} \gamma_{2}+\frac{2}{3} \gamma_{3} .
$$

Then, solutions of (33) are defined as follows:

$$
\begin{array}{r}
u(x, t)=\phi(\xi) \exp (i(\lambda x-\omega t)), \quad \xi=k(x-c t), \\
\omega=\frac{\left(\alpha-\gamma_{2} \lambda\right)\left(2 \lambda-c-3 \gamma_{1} \lambda^{2}\right)}{C}+\lambda^{2}-\gamma_{1} \lambda^{3}, \\
\lambda=\frac{C-\alpha \lambda_{1}}{3 C \gamma_{1}-\gamma_{1} \gamma_{2}},
\end{array}
$$

where $\phi(\xi)$, appearing in these solutions, is given by relations (12), (15), and (28)-(32). $A, B$, and $C$ are defined by (42).

\subsection{The Klein-Gordon-Zakharov (KGZ) System [21].} Consider

$$
\begin{gathered}
E_{t t}-E_{x x}+E-\alpha N E-\beta|E|^{2} E=0, \\
N_{t t}-N_{x x}=\gamma\left(|E|^{2}\right)_{x x}
\end{gathered}
$$

wherein the complex valued unknown function $E=E(x, t)$ denotes the fast time scale component of electric field raised by electrons, and the real valued unknown function $N=$ $N(x, t)$ represents the deviation of ion density. $\alpha, \beta$ and $\gamma$ are some real parameters.

We assume that

$$
E(x, t)=\phi(\xi) \exp (i(k x+\omega t)), \quad \begin{aligned}
& N=N(\xi), \\
& \xi=\omega x+k t .
\end{aligned}
$$

Substituting (45) into (44), we have

$$
\begin{gathered}
-\alpha N \phi-\beta \phi^{3}+\left(k^{2}-\omega^{2}+1\right) \phi+\left(k^{2}-\omega^{2}\right) \phi^{\prime \prime}=0, \\
\left(k^{2}-\omega^{2}\right) N^{\prime \prime}-\gamma \omega^{2}\left(2 \phi^{\prime 2}+2 \phi \phi^{\prime \prime}\right)=0 .
\end{gathered}
$$

Integrating (47) with respect to $\xi$ twice and setting the integration constant to be zero, then we have

$$
N=\frac{\gamma \omega^{2}}{k^{2}-\omega^{2}} \phi^{2}
$$

Substituting (48) into (46), we have

$$
A \phi^{\prime \prime}(\xi)+B \phi(\xi)+C \phi^{3}(\xi)=0 .
$$

Equation (49) is identical to (9) and $A, B$, and $C$ are defined by

$$
\begin{gathered}
A=k^{2}-\omega^{2}, \quad B=k^{2}-\omega^{2}+1, \\
C=-\left(\beta+\frac{\alpha \gamma \omega^{2}}{k^{2}-\omega^{2}}\right) .
\end{gathered}
$$

Then, solutions of the Klein-Gordon-Zakharov (KGZ) system are defined as follows:

$$
\begin{array}{r}
E(x, t)=\phi(\xi) \exp (i(k x+\omega t)), \quad N=\frac{\gamma \omega^{2}}{k^{2}-\omega^{2}} \phi^{2}, \\
\xi=\omega x+k t,
\end{array}
$$

where $\phi(\xi)$ appearing in these solutions is given by relations (12), (15), and (28)-(32). A, B, and $C$ are defined by (50).

4.3. A Class of Nonlinear Partial Differential Equations (NPDEs). We consider a class of NLPDEs with constant coefficients [22]

$$
\begin{gathered}
i u_{t}+\mu\left(u_{x x}+D_{1} u_{y y}\right)+E_{1}|u|^{2} u+A_{1} u n=0, \\
D_{2} n_{t t}+\left(n_{x x}-E_{2} u_{y y}\right)+A_{2}\left(|u|^{2}\right)_{x x}=0,
\end{gathered}
$$

where $\mu, D_{i}, E_{i}$, and $A_{i}(i=1,2)$ are real constants and $\mu \neq 0, D_{1} \neq 0, A_{1} \neq 0$, and $A_{2} \neq 0$. Equations (52) are a class of physically important equations. In fact, if one takes

$$
\begin{array}{llll}
\mu=\frac{1}{2} \kappa^{2}, & D_{1}=2 \mu, & E_{1}=\alpha, & A_{1}=-1, \\
D_{2}=0, & E_{2}=D_{1}, & A_{2}=-2 \alpha, & \kappa^{2}= \pm 1,
\end{array}
$$

then (52) represent the Davey-Stewartson (DS) equations [23]

$$
\begin{gathered}
i u_{t}+\frac{1}{2} \kappa^{2}\left(u_{x x}+\kappa^{2} u_{y y}\right)+\alpha|u|^{2} u-u n=0, \\
n_{x x}-\kappa^{2} n_{y y}-2 \alpha\left(|u|^{2}\right)_{x x}=0 .
\end{gathered}
$$

If one takes

$$
\begin{aligned}
& n=n(x, t), \quad \text { that is, } n_{y}=0, \quad \mu=1, \\
& D_{1}=0, \quad E_{1}=-2 \lambda, \\
& E_{2}=-1, \quad A_{2}=-1, \quad A_{1}=2,
\end{aligned}
$$

then (52) become generalized Zakharov (GZ) equations [24]

$$
\begin{gathered}
i u_{t}+u_{x x}-2 \lambda|u|^{2} u+2 u n=0, \\
n_{t t}-n_{x x}+\left(|u|^{2}\right)_{x x}=0 .
\end{gathered}
$$

Since $u$ is a complex function, we assume that

$$
\begin{array}{r}
u(x, t)=\phi(\xi) \exp (i(k x+l y-\Omega t)), \\
n=n(\xi) \quad \xi=p x+q y-\omega t,
\end{array}
$$


where both $\phi(\xi)$ and $n(\xi)$ are real functions and $k, l, p$, $q$, $\Omega$, and $\omega$ are constants to be determined later. Substituting (57) into (52), we have the following ODE for $\phi(\xi)$ and $n(\xi)$ :

$$
\begin{aligned}
& E_{1} \phi^{3}+\left(\Omega-\mu\left(k^{2}+D_{1} l^{2}\right)+A_{1} n\right) \phi+\mu\left(D_{1} q^{2}+p^{2}\right) \phi^{\prime \prime} \\
&+i\left(2 \mu\left(p k+D_{1} q l\right)-\omega\right) \phi^{\prime}=0 \\
&\left(D_{2} \omega^{2}+p^{2}-E_{2} q^{2}\right) n^{\prime \prime}+A_{2} p^{2}\left(2 \phi^{\prime 2}+2 \phi \phi^{\prime \prime}\right)=0
\end{aligned}
$$

If we set

$$
\omega=2 \mu\left(p k+D_{1} q l\right),
$$

then $(58)$ reduces to

$$
\begin{aligned}
E_{1} \phi^{3} & +\left(\Omega-\mu\left(k^{2}+D_{1} l^{2}\right)+A_{1} n\right) \phi \\
& +\mu\left(D_{1} q^{2}+p^{2}\right) \phi^{\prime \prime}=0 .
\end{aligned}
$$

Integrating (59) twice to $\xi$, we get

$$
n=\frac{D}{D_{2} \omega^{2}+p^{2}-E_{2} q^{2}}-\frac{A_{2} p^{2}}{D_{2} \omega^{2}+p^{2}-E_{2} q^{2}} \phi^{2},
$$

where $D$ is the integrating constant and Substituting (62) into (61) yields

$$
A \phi^{\prime \prime}+B \phi+C \phi^{3}=0
$$

Equation (63) is identical to (9) and $A, B$, and $C$ are defined by

$$
\begin{gathered}
A=\mu\left(D_{1} q^{2}+p^{2}\right), \\
B=\Omega-\mu\left(k^{2}+D_{1} l^{2}\right)+\frac{A_{1} D}{D_{2} \omega^{2}+p^{2}-E_{2} q^{2}}, \\
C=E_{1}-\frac{A_{1} A_{2} p^{2}}{D_{2} \omega^{2}+p^{2}-E_{2} q^{2}} .
\end{gathered}
$$

Then, solutions of (52) are defined as follows:

$$
\begin{gathered}
u(x, y, t)=\phi(\xi) \exp (i(k x+l y-\Omega t)), \\
\xi=p x+q y-\omega t, \quad \omega=2 \mu\left(p k+D_{1} q l\right), \\
n(x, y, t)=\frac{D}{D_{2} \omega^{2}+p^{2}-E_{2} q^{2}}-\frac{A_{2} p^{2}}{D_{2} \omega^{2}+p^{2}-E_{2} q^{2}} \phi^{2},
\end{gathered}
$$

where $\phi(\xi)$ appearing in these solutions is given by relations (12), (15), and (28)-(32) and $A, B$, and $C$ are defined by (64).

We may obtain from (54) that

$$
\begin{gathered}
\omega=\kappa^{2}\left(p k+\kappa^{2} q l\right), \\
u(x, y, t)=\phi(\xi) \exp (i(k x+l y-\Omega t)), \\
n(x, y, t)=\frac{D}{\omega^{2}-\kappa^{2} q^{2}}+\frac{2 \alpha p^{2}}{\omega^{2}-\kappa^{2} q^{2}} \phi^{2},
\end{gathered}
$$

where $D$ is the integrating constant and then (54) reduce to

$$
A \phi^{\prime \prime}+B \phi+C \phi^{3}=0
$$

This equation coincides also with (9), where $A, B$, and $C$ are defined as follows:

$$
\begin{gathered}
A=\frac{1}{2} \kappa^{2}\left(p^{2}+\kappa^{2} q^{2}\right) \\
B=\Omega-\frac{1}{2} \kappa^{2}\left(k^{2}+\kappa^{2} l^{2}\right)-\frac{D}{\omega^{2}-\kappa^{2} q^{2}} \\
C=\alpha-\frac{2 \alpha p^{2}}{\omega^{2}-\kappa^{2} q^{2}}
\end{gathered}
$$

where $\xi=p x+q y-\omega t, \phi(\xi)$ appearing in these solutions is given by relations (12), (15), and (28)-(32) and $A, B$, and $C$ are defined by (68).

We may obtain from (56) that

$$
\begin{gathered}
\omega=2 p k, \\
u(x, t)=\phi(\xi) \exp (i(k x-\Omega t)), \\
n(x, t)=\frac{D}{p^{2}-\omega^{2}}+\frac{p^{2}}{p^{2}-\omega^{2}} \phi^{2},
\end{gathered}
$$

where $D$ is the integrating constant and then (56) reduce to

$$
A \phi^{\prime \prime}+B \phi+C \phi^{3}=0
$$

This equation coincides also with (9), where $A, B$, and $C$ are defined as follows:

$$
\begin{gathered}
A=p^{2}, \quad B=\Omega-k^{2}+\frac{2 D}{p^{2}-\omega^{2}}, \\
C=2\left(\frac{p^{2}}{p^{2}-\omega^{2}}-\lambda\right),
\end{gathered}
$$

where $\xi=p x-\omega t, \phi(\xi)$ appearing in these solutions is given by relations (12), (15), and (28)-(32) and $A, B$, and $C$ are defined by (71).

\section{Conclusions}

The simplest equation method is a very powerful mathematical technique for finding exact solutions of nonlinear ordinary differential equations, and the elliptic-like equation is one of the most important auxiliary equations because many nonlinear evolution equations, such as the perturbed nonlinear Schrödinger's equation, the Klein-Gordon-Zakharov system, the generalized Davey-Stewartson equations, the DaveyStewartson equations, the generalized Zakharov equations, the Hamilton amplitude equation, the generalized HirotaSatsuma coupled KdV system, and the generalized ZK$\mathrm{BBM}$ equation, can be converted to this equation using the travelling wave reduction.

In this paper, we apply the simplest equation method and the modified simplest equation method to derive the exact solutions of the elliptic-like equation. The exact solutions of 
the perturbed nonlinear Schrödinger's equation, the KleinGordon-Zakharov system, the generalized Davey-Stewartson equations, the Davey-Stewartson equations, and the generalized Zakharov equations are derived. Comparing the currently proposed method with other methods, such as the $\left(G^{\prime} / G\right)$-expansion method, the various extended hyperbolic methods, and the exp-function method, we might conclude that some exact solutions that we obtained can be investigated using these methods with the aid of the symbolic computation software, such as Matlab, Mathematica, and Maple to facilitate the complicated algebraic computations. But, by means of the simplest equation method and the modified simplest equation method the exact solutions to these equations have been gained in this paper without using the symbolic computation software since the computations are simple. This study shows that the simplest equation method and the modified simplest equation method are much more simple than the other methods and can be applied to many other nonlinear evolution equations.

\section{Conflict of Interests}

The authors declare that there is no conflict of interests regarding the publication of this paper.

\section{Acknowledgments}

This work was supported by the National Natural Science Foundation of China (11161020; 11361023), the Natural Science Foundation of Yunnan Province (2011FZ193; 2013FZ117), and the Natural Science Foundation of Education Committee of Yunnan Province (2012Y452; 2013C079).

\section{References}

[1] M. L. Wang, "Exact solutions for a compound KdV-Burgers equation," Physics Letters A, vol. 213, no. 5-6, pp. 279-287, 1996.

[2] M. L. Wang, Y. B. Zhou, and Z. B. Li, "Application of a homogeneous balance method to exact solutions of nonlinear equations in mathematical physics," Physics Letters A, vol. 216, no. $1-5$, pp. 67-75, 1996.

[3] Sirendaoreji and S. Jiong, "Auxiliary equation method for solving nonlinear partial differential equations," Physics Letters A, vol. 309, no. 5-6, pp. 387-396, 2003.

[4] Z. Y. Yan and H. Q. Zhang, "New explicit and exact travelling wave solutions for a system of variant Boussinesq equations in mathematical physics," Physics Letters A, vol. 252, no. 6, pp. 291296, 1999.

[5] E. Fan and J. Zhang, "Applications of the Jacobi elliptic function method to special-type nonlinear equations," Physics Letters A, vol. 305, no. 6, pp. 383-392, 2002.

[6] X.-H. Wu and J.-H. He, "EXP-function method and its application to nonlinear equations," Chaos, Solitons \& Fractals, vol. 38, no. 3, pp. 903-910, 2008.

[7] H. A. Abdusalam, "On an improved complex tanh-function method," International Journal of Nonlinear Sciences and Numerical Simulation, vol. 6, no. 2, pp. 99-106, 2005.

[8] A. A. Soliman, "Extended improved tanh-function method for solving the nonlinear physical problems," Acta Applicandae Mathematicae, vol. 104, no. 3, pp. 367-383, 2008.
[9] S. B. Leble and N. V. Ustinov, "Darboux transforms, deep reductions and solitons," Journal of Physics A, vol. 26, no. 19, pp. 5007-5016, 1993

[10] H.-C. Hu, X.-Y. Tang, S.-Y. Lou, and Q.-P. Liu, "Variable separation solutions obtained from Darboux transformations for the asymmetric Nizhnik-Novikov-Veselov system," Chaos, Solitons \& Fractals, vol. 22, no. 2, pp. 327-334, 2004.

[11] M. L. Wang, X. Z. Li, and J. L. Zhang, “The $\left(G^{\prime} / G\right)$-expansion method and travelling wave solutions of nonlinear evolution equations in mathematical physics," Physics Letters A, vol. 372, no. 4, pp. 417-423, 2008.

[12] S. M. Guo and Y. B. Zhou, "The extended $\left(G^{\prime} / G\right)$-expansion method and its applications to the Whitham-Broer-Kauplike equations and coupled Hirota-Satsuma KdV equations," Applied Mathematics and Computation, vol. 215, no. 9, pp. 32143221, 2010.

[13] N. A. Kudryashov, "Exact solitary waves of the Fisher equation," Physics Letters A, vol. 342, no. 1-2, pp. 99-106, 2005.

[14] N. A. Kudryashov, "Simplest equation method to look for exact solutions of nonlinear differential equations," Chaos, Solitons and Fractals, vol. 24, no. 5, pp. 1217-1231, 2005.

[15] N. K. Vitanov and Z. I. Dimitrova, "Application of the method of simplest equation for obtaining exact traveling-wave solutions for two classes of model PDEs from ecology and population dynamics," Communications in Nonlinear Science and Numerical Simulation, vol. 15, no. 10, pp. 2836-2845, 2010.

[16] E. M. E. Zayed and S. A. Hoda Ibrahim, "Exact solutions of nonlinear evolution equations in mathematical physics using the modified simple equation method," Chinese Physics Letters, vol. 29, no. 6, Article ID 060201, 2012.

[17] A. J. Mohamad Jawad, M. D. Petković, and A. Biswas, "Modified simple equation method for nonlinear evolution equations," Applied Mathematics and Computation, vol. 217, no. 2, pp. 869$877,2010$.

[18] A. Yildirim, A. Samiei Paghaleh, M. Mirzazadeh, H. Moosaei, and A. Biswas, "New exact traveling wave solutions for DS-I and DS-II equations," Nonlinear Analysis: Modelling and Control, vol. 17, no. 3, pp. 369-378, 2012.

[19] N. Taghizadeh, M. Mirzazadeh, A. Samiei Paghaleh, and J. Vahidi, "Exact solutions of nonlinear evolution equations by using the modified simple equation method," Ain Shams Engineering Journal, vol. 3, no. 3, pp. 321-325, 2012.

[20] Z.-Y. Zhang, Z.-H. Liu, X.-J. Miao, and Y.-Z. Chen, "New exact solutions to the perturbed nonlinear Schrödinger's equation with Kerr law nonlinearity," Applied Mathematics and Computation, vol. 216, no. 10, pp. 3064-3072, 2010.

[21] Q. H. Shi, Q. Xiao, and X. J. Liu, "Extended wave solutions for a nonlinear Klein-Gordon-Zakharov system," Applied Mathematics and Computation, vol. 218, no. 19, pp. 9922-9929, 2012.

[22] Y. B. Zhou, M. L. Wang, and T. D. Miao, "The periodic wave solutions and solitary wave solutions for a class of nonlinear partial differential equations," Physics Letters A, vol. 323, no. 1-2, pp. 77-88, 2004.

[23] A. Davey and K. Stewartson, "On three-dimensional packets of surface waves," Proceedings of the Royal Society A, vol. 338, pp. 101-110, 1974.

[24] B. Malomed, D. Anderson, M. Lisak, M. L. Quiroga-Teixeiro, and L. Stenflo, "Dynamics of solitary waves in the Zakharov model equations," Physical Review E, vol. 55, no. 1, pp. 962-968, 1997. 


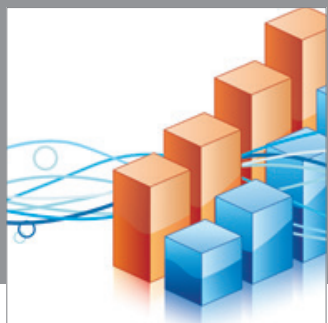

Advances in

Operations Research

mansans

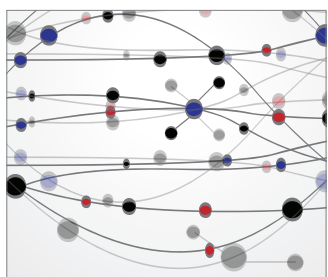

The Scientific World Journal
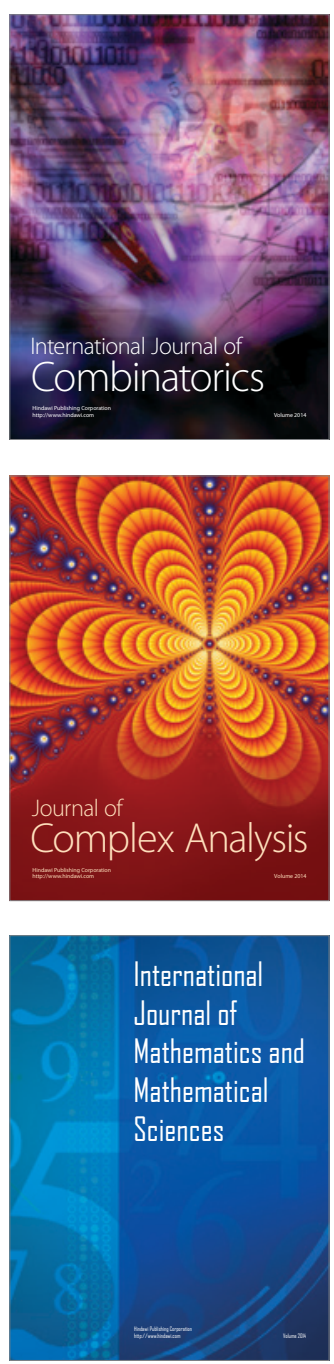
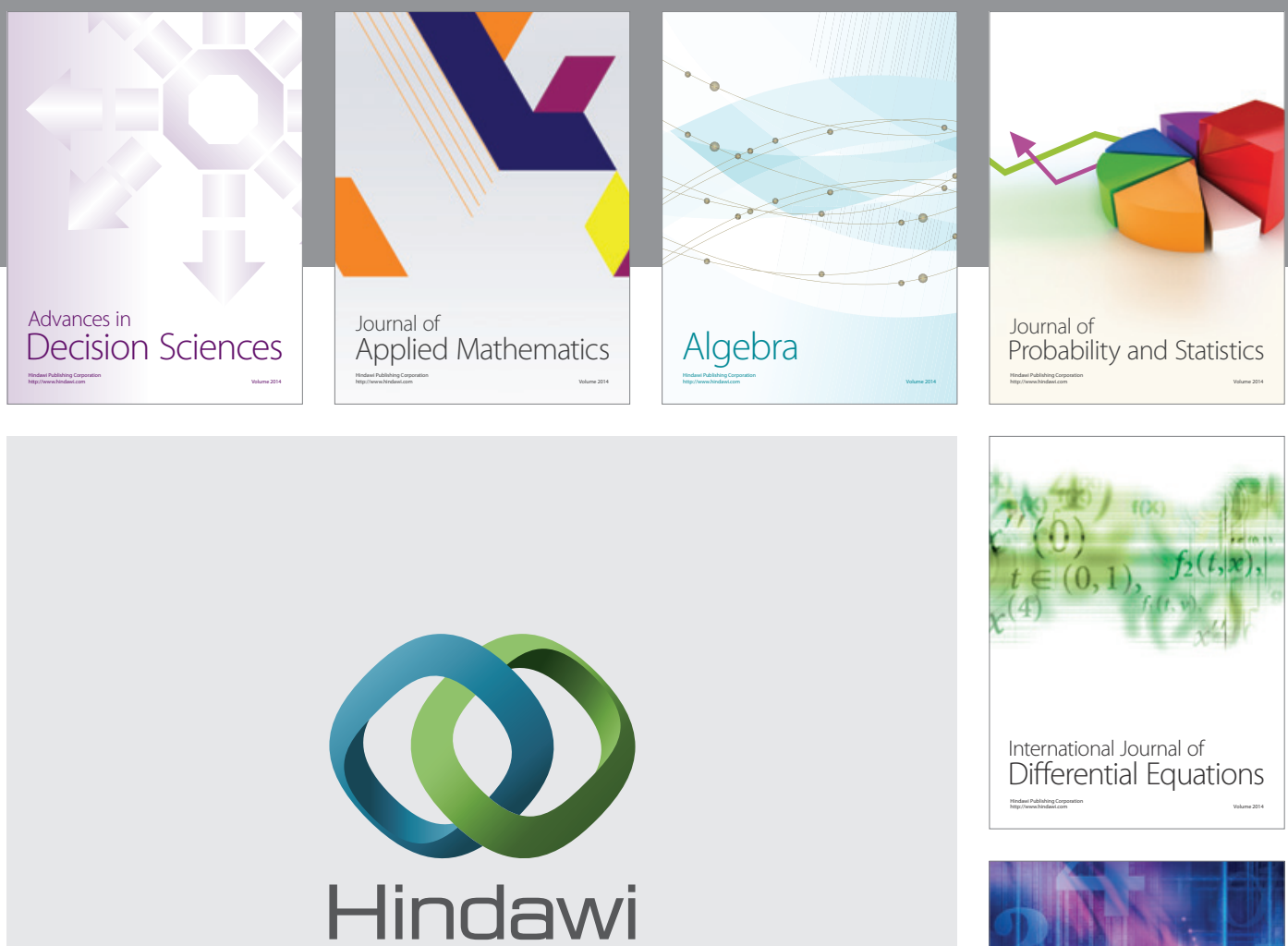

Submit your manuscripts at http://www.hindawi.com
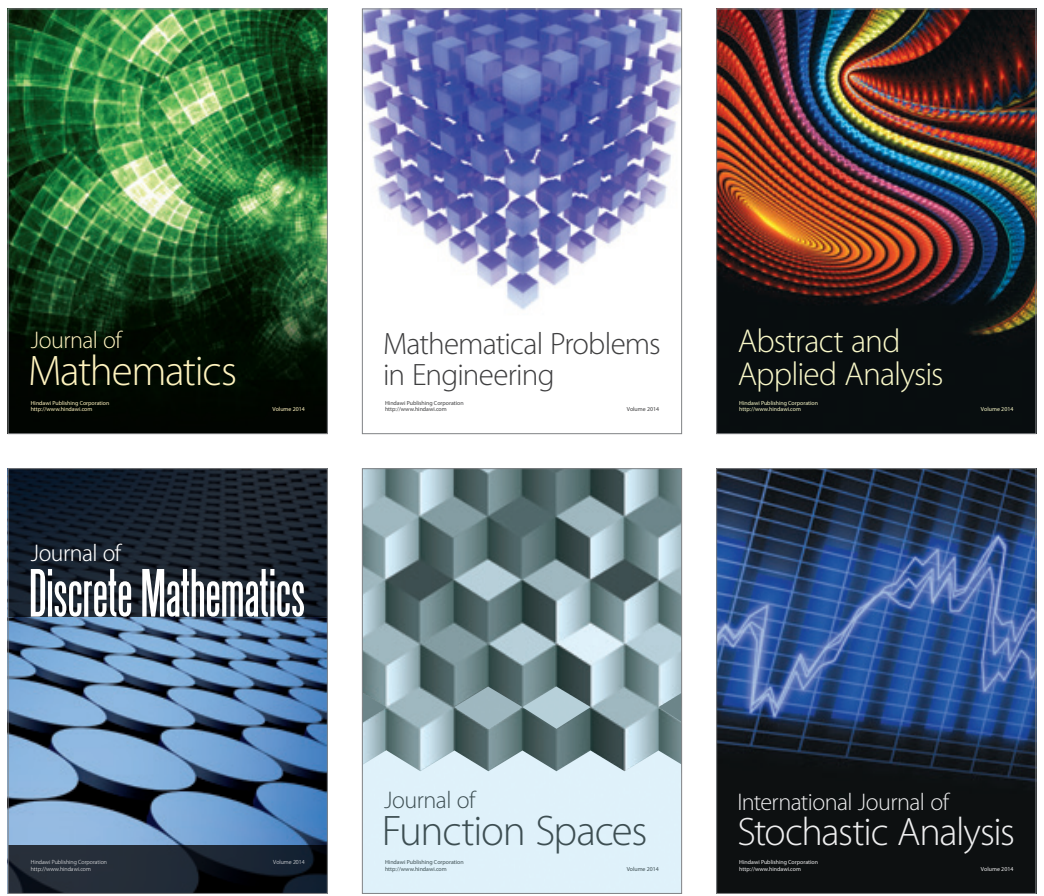

Journal of

Function Spaces

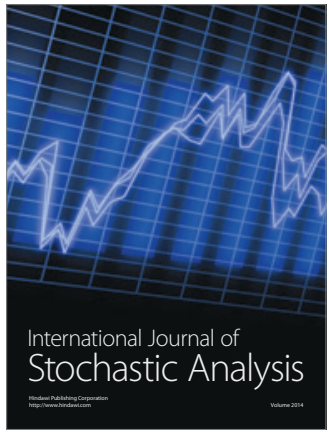

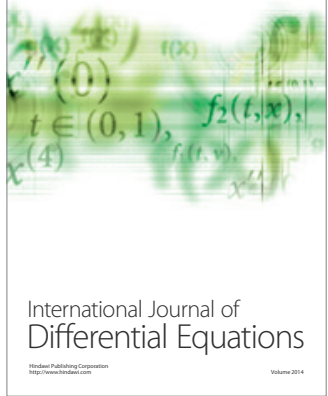
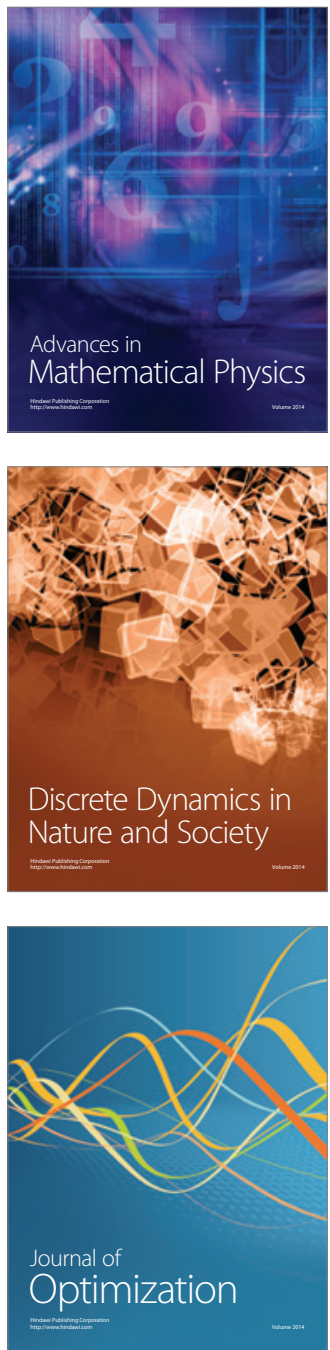